\title{
Status of Advanced Virgo
}

F. Acernese 51,17 , T. Adams ${ }^{26}$, K. Agatsuma ${ }^{31}$, L. Aiello ${ }^{10,16}$, A. Allocca ${ }^{47,21, \text {,a A. Amato }}{ }^{28}$, S. Antier ${ }^{25}$, N. Arnaud ${ }^{25,8}$, S. Ascenzi ${ }^{50,23}$, P. Astone ${ }^{22}$, P. Bacon ${ }^{1}$, M. K. M. Bader ${ }^{31}$, F. Baldaccini ${ }^{46,20}$, G. Ballardin ${ }^{8}$, F. Barone ${ }^{51,17}$, M. Barsuglia ${ }^{1}$, D. Barta ${ }^{34}$, A. Basti ${ }^{47,21}$, M. Bawaj ${ }^{38,20}$, M. Bazzan ${ }^{44,18}$, M. Bejger ${ }^{5}$, I. Belahcene ${ }^{25}$, D. Bersanetti ${ }^{15}$, A. Bertolini ${ }^{31}$, M. Bitossi ${ }^{8,21}$, M. A. Bizouard ${ }^{25}$, S. Bloemen ${ }^{33}$, M. Boer ${ }^{2}$, G. Bogaert ${ }^{2}$, F. Bondu ${ }^{48}$, R. Bonnand ${ }^{26}$, B. A. Boom ${ }^{31}$, V. Boschi ${ }^{8,21}$, Y. Bouffanais ${ }^{1}$, A. Bozzi ${ }^{8}$, C. Bradaschia ${ }^{21}$, M. Branchesi ${ }^{10,16}$, T. Briant ${ }^{27}$, A. Brillet ${ }^{2}$, V. Brisson ${ }^{25}$, T. Bulik ${ }^{3}$, H. J. Bulten ${ }^{36,31}$, D. Buskulic ${ }^{26}$, C. Buy ${ }^{1}$, G. Cagnoli ${ }^{28,42}$, E. Calloni ${ }^{43,17}$, M. Canepa ${ }^{39,15}$, P. Canizares ${ }^{33}$, E. Capocasa ${ }^{1}$, F. Carbognani ${ }^{8}$, J. Casanueva Diaz ${ }^{21}$, C. Casentini ${ }^{50,23}$, S. Caudill ${ }^{31}$, F. Cavalier ${ }^{25}$, R. Cavalieri ${ }^{8}$, G. Cella ${ }^{21}$, P. Cerdá-Durán ${ }^{55}$, G. Cerretani ${ }^{47,21}$, E. Cesarini ${ }^{6,23}$, E. Chassande-Mottin ${ }^{1}$, A. Chincarini ${ }^{15}$, A. Chiummo ${ }^{8}$, N. Christensen ${ }^{2}$, S. Chua ${ }^{27}$, G. Ciani ${ }^{44,18}$, R. Ciolfi ${ }^{13,24}$, A. Cirone ${ }^{39,15}$, F. Cleva ${ }^{2}$, E. Coccia ${ }^{10,16}$, P.-F. Cohadon ${ }^{27}$, D. Cohen ${ }^{25}$, A. Colla ${ }^{49,22}$, L. Conti ${ }^{18}$, I. Cordero-Carrión ${ }^{56}$, S. Cortese ${ }^{8}$, J.-P. Coulon' ${ }^{2}$, E. Cuoco ${ }^{8}$, S. D'Antonio ${ }^{23}$, V. Dattilo ${ }^{8}$, M. Davier ${ }^{25}$, C. De Rossi ${ }^{28,8}$, J. Degallaix ${ }^{28}$, M. De Laurentis ${ }^{10,17}$, S. Deléglise ${ }^{27}$, W. Del Pozzo ${ }^{47,21}$, R. De Pietri ${ }^{45,19}$, R. De Rosa ${ }^{43,17}$, L. Di Fiore ${ }^{17}$, M. Di Giovanni ${ }^{33,24}$, T. Di Girolamo43,17, A. Di Lieto ${ }^{47,21}$, S. Di Pace ${ }^{49,22}$, I. Di Palma ${ }^{49,22}$, F. Di Renzo ${ }^{47,21}$, V. Dolique ${ }^{28}$, M. Ducrot ${ }^{26}$, D. Estevez ${ }^{26}$, V. Fafone ${ }^{50,23,10}$, S. Farinon ${ }^{15}$, I. Ferrante ${ }^{47,21}$, F. Ferrini ${ }^{8}$, F. Fidecaro ${ }^{47,21}$, I. Fiori ${ }^{8}$, D. Fiorucci ${ }^{1}$, R. Flaminio ${ }^{28,29}$, J. A. Font ${ }^{55,32}$, J.-D. Fournier ${ }^{2}$, S. Frasca ${ }^{49,22}$, F. Frasconi ${ }^{21}$, V. Frey ${ }^{25}$, L. Gammaitoni ${ }^{46}$, F. Garufi ${ }^{43,17}$, G. Gemme ${ }^{15}$, E. Genin ${ }^{8}$, A. Gennai ${ }^{21}$, V. Germain ${ }^{26}$, Archisman Ghosh $^{31}$, S. Ghosh ${ }^{33,31}$, A. Giazotto ${ }^{21}$, J. M. Gonzalez Castro ${ }^{47,21}$, M. Gosselin ${ }^{8}$, R. Gouaty ${ }^{26}$, A. Grado ${ }^{12,17}$, M. Granata ${ }^{28}$, G. Greco ${ }^{54,14}$, P. Groot ${ }^{33}$, P. Gruning ${ }^{25}$, G. M. Guidi ${ }^{54,14}$, O. Halim ${ }^{16,10}$, J. Harms ${ }^{10,16}$, A. Heidmann ${ }^{27}$, H. Heitmann ${ }^{2}$, P. Hello ${ }^{25}$, G. Hemming ${ }^{8}$, T. Hinderer ${ }^{33}$, D. Hoak ${ }^{8}$, D. Hofman'28, A. Hreibi², D. Huet ${ }^{25}$, G. Intini ${ }^{49,22}$, J.-M. Isac ${ }^{27}$, T. Jacqmin ${ }^{27}$, P. Jaranowski ${ }^{37}$, R. J. G. Jonker ${ }^{31}$, F. Kéfélian ${ }^{2}$, I. Khan ${ }^{10,23}$, S. Koley ${ }^{31}$, I. Kowalska ${ }^{3}$, A. Królak ${ }^{30,11}$, A. Kutynia ${ }^{30}$, A. Lartaux-Vollard ${ }^{25}$, C. Lazzaro $^{18}$, P. Leaci ${ }^{49,22}$, M. Leonardi ${ }^{53,24}$, N. Leroy ${ }^{25}$, N. Letendre ${ }^{26}$, M. Lorenzini ${ }^{10,16}$, V. Loriette ${ }^{9}$, G. Losurdo ${ }^{21}$, D. Lumaca ${ }^{50,23}$, E. Majorana ${ }^{22}$, I. Maksimovic ${ }^{9}$, N. Man $^{2}$, M. Mantovani ${ }^{8}$, F. Marchesoni ${ }^{38,20}$, F. Marion ${ }^{26}$, A. Marquina ${ }^{56}$, F. Martelli ${ }^{54,14}$, L. Martellini ${ }^{2}$, A. Masserot ${ }^{26}$, S. Mastrogiovanni' ${ }^{49,22}$, J. Meidam ${ }^{31}$, M. Merzougui ${ }^{2}$, R. Metzdorff ${ }^{27}$, C. Michel ${ }^{28}$, L. Milano ${ }^{43,17}$, A. Miller ${ }^{49,22}$, O. Minazzoli2,7, Y. Minenkov ${ }^{23}$, A. Moggi ${ }^{21}$, M. Mohan ${ }^{8}$, M. Montani ${ }^{54,14}$, B. Mours ${ }^{26}$, I. Nardecchia ${ }^{50,23}$, L. Naticchioni ${ }^{49,22}$, G. Nelemans ${ }^{33,31}$, D. Nichols ${ }^{33}$, S. Nissanke ${ }^{33,31}$, F. Nocera ${ }^{8}$, C. Palomba ${ }^{22}$, F. Paoletti ${ }^{21}$, A. Paoli ${ }^{8}$, A. Pasqualetti ${ }^{8}$, R. Passaquieti ${ }^{47,21}$, D. Passuello ${ }^{21}$, M. Patil ${ }^{11}$, B. Patricelli ${ }^{35,21}$, R. Pedurand ${ }^{28,41}$, A. Perreca ${ }^{53,24}$, O. J. Piccinni ${ }^{49,22}$, M. Pichot ${ }^{2}$, F. Piergiovanni ${ }^{54,14}$, G. Pillant ${ }^{8}$, L. Pinard ${ }^{28}$, R. Poggiani ${ }^{47,21}$, P. Popolizio ${ }^{8}$, E. K. Porter ${ }^{1}$, G. A. Prodi ${ }^{53,24}$, M. Punturo ${ }^{20}$, P. Puppo ${ }^{22}$, P. Rapagnani ${ }^{49,22}$, M. Razzano ${ }^{47,21}$, T. Regimbau ${ }^{2}$, L. Rei ${ }^{15}$, F. Ricci ${ }^{49,22}$, F. Robinet ${ }^{25}$, A. Rocchi ${ }^{23}$, L. Rolland ${ }^{26}$, R. Romano ${ }^{51,17}$, D. Rosińska ${ }^{4,5}$, P. Ruggi ${ }^{8}$, L. Salconi ${ }^{8}$, N. Sanchis-Gual ${ }^{55}$, B. Sassolas ${ }^{28}$, P. Schmidt ${ }^{33}$, D. Sentenac ${ }^{8}$, V. Sequino ${ }^{50,23,10}$, M. Sieniawska ${ }^{5}$, A. Singhal ${ }^{10,22}$,

\footnotetext{
ae-mail: allocca@pi.infn.it
} 
F. Sorrentino ${ }^{15}$, G. Stratta ${ }^{54,14}$, B. L. Swinkels ${ }^{8}$, M. Tacca ${ }^{31}$, S. Tiwari ${ }^{10,24}$, M. Tonelli ${ }^{47,21}$, A. TorresForné $^{55}$, F. Travasso ${ }^{8,20}$, M. C. Tringali ${ }^{53,24}$, L. Trozzo ${ }^{52,21}$, K. W. Tsang ${ }^{31}$, N. van Bakel ${ }^{31}$, M. van Beuzekom ${ }^{31}$, J. F. J. van den Brand ${ }^{36,31}$, C. Van Den Broeck ${ }^{31,40}$, L. van der Schaaf ${ }^{31}$, J. V. van Heijningen ${ }^{31}$, M. Vardaro ${ }^{44,18}$, M. Vasúth ${ }^{34}$, G. Vedovato ${ }^{18}$, D. Verkindt ${ }^{26}$, F. Vetrano ${ }^{54,14}$,

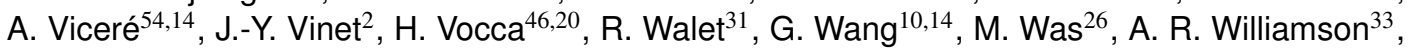
M. Yvert ${ }^{26}$, A. Zadrożny ${ }^{30}$, T. Zelenova ${ }^{8}$, J.-P. Zendri ${ }^{18}$

${ }^{1}$ APC, AstroParticule et Cosmologie, Université Paris Diderot, CNRS/IN2P3, CEA/Irfu, Observatoire de Paris, Sorbonne Paris Cité, F-75205 Paris Cedex 13, France,

${ }^{2}$ Artemis, Université Côte d'Azur, Observatoire Côte d'Azur, CNRS, CS 34229, F-06304 Nice Cedex 4, France,

${ }^{3}$ Astronomical Observatory Warsaw University, 00-478 Warsaw, Poland,

${ }^{4}$ Janusz Gil Institute of Astronomy, University of Zielona Góra, 65-265 Zielona Góra, Poland,

${ }^{5}$ Nicolaus Copernicus Astronomical Center, Polish Academy of Sciences, 00-716, Warsaw, Poland,

${ }^{6}$ Museo Storico della Fisica e Centro Studi e Ricerche Enrico Fermi, I-00184 Roma, Italy,

${ }^{7}$ Centre Scientifique de Monaco, 8 quai Antoine ler, MC-98000, Monaco,

${ }^{8}$ European Gravitational Observatory (EGO), I-56021 Cascina, Pisa, Italy,

${ }^{9} \mathrm{ESPCl}, \mathrm{CNRS}, \mathrm{F}-75005$ Paris, France,

${ }^{10}$ Gran Sasso Science Institute (GSSI), I-67100 L'Aquila, Italy,

${ }^{11}$ Institute of Mathematics, Polish Academy of Sciences, 00656 Warsaw, Poland,

${ }^{12}$ INAF, Osservatorio Astronomico di Capodimonte, I-80131, Napoli, Italy,

${ }^{13}$ INAF, Osservatorio Astronomico di Padova, I-35122 Padova, Italy,

${ }^{14}$ INFN, Sezione di Firenze, I-50019 Sesto Fiorentino, Firenze, Italy,

${ }^{15}$ INFN, Sezione di Genova, I-16146 Genova, Italy,

${ }^{16}$ INFN, Laboratori Nazionali del Gran Sasso, I-67100 Assergi, Italy,

${ }^{17}$ INFN, Sezione di Napoli, Complesso Universitario di Monte S.Angelo, I-80126 Napoli, Italy,

${ }^{18}$ INFN, Sezione di Padova, I-35131 Padova, Italy,

${ }^{19}$ INFN, Sezione di Milano Bicocca, Gruppo Collegato di Parma, I-43124 Parma, Italy,

${ }^{20}$ INFN, Sezione di Perugia, I-06123 Perugia, Italy,

${ }^{21}$ INFN, Sezione di Pisa, I-56127 Pisa, Italy,

${ }^{22}$ INFN, Sezione di Roma, I-00185 Roma, Italy,

${ }^{23}$ INFN, Sezione di Roma Tor Vergata, I-00133 Roma, Italy,

${ }^{24}$ INFN, Trento Institute for Fundamental Physics and Applications, I-38123 Povo, Trento, Italy,

${ }^{25}$ LAL, Univ. Paris-Sud, CNRS/IN2P3, Université Paris-Saclay, F-91898 Orsay, France,

${ }^{26}$ Laboratoire d'Annecy-le-Vieux de Physique des Particules (LAPP), Université Savoie Mont Blanc, CNRS/IN2P3, F-74941 Annecy, France,

${ }^{27}$ Laboratoire Kastler Brossel, UPMC-Sorbonne Universités, CNRS, ENS-PSL Research University, Collège de France, F-75005 Paris, France,

${ }^{28}$ Laboratoire des Matériaux Avancés (LMA), CNRS/IN2P3, F-69622 Villeurbanne, France,

${ }^{29}$ National Astronomical Observatory of Japan, 2-21-1 Osawa, Mitaka, Tokyo 181-8588, Japan,

${ }^{30}$ NCBJ, 05-400 Świerk-Otwock, Poland,

${ }^{31}$ Nikhef, Science Park, 1098 XG Amsterdam, The Netherlands,

${ }^{32}$ Observatori Astronòmic, Universitat de València, E-46980 Paterna, València, Spain,

${ }^{33}$ Department of Astrophysics/IMAPP, Radboud University Nijmegen, P.O. Box 9010, 6500 GL Nijmegen, The Netherlands,

${ }^{34}$ Wigner RCP, RMKI, H-1121 Budapest, Konkoly Thege Miklós út 29-33, Hungary,

${ }^{35}$ Scuola Normale Superiore, Piazza dei Cavalieri 7, I-56126 Pisa, Italy,

${ }^{36}$ VU University Amsterdam, 1081 HV Amsterdam, The Netherlands,

${ }^{37}$ University of Białystok, 15-424 Białystok, Poland,

${ }^{38}$ Università di Camerino, Dipartimento di Fisica, I-62032 Camerino, Italy,

${ }^{39}$ Dipartimento di Fisica, Università degli Studi di Genova, I-16146 Genova, Italy,

${ }^{40}$ Van Swinderen Institute for Particle Physics and Gravity, University of Groningen, Nijenborgh 4, 9747 AG 


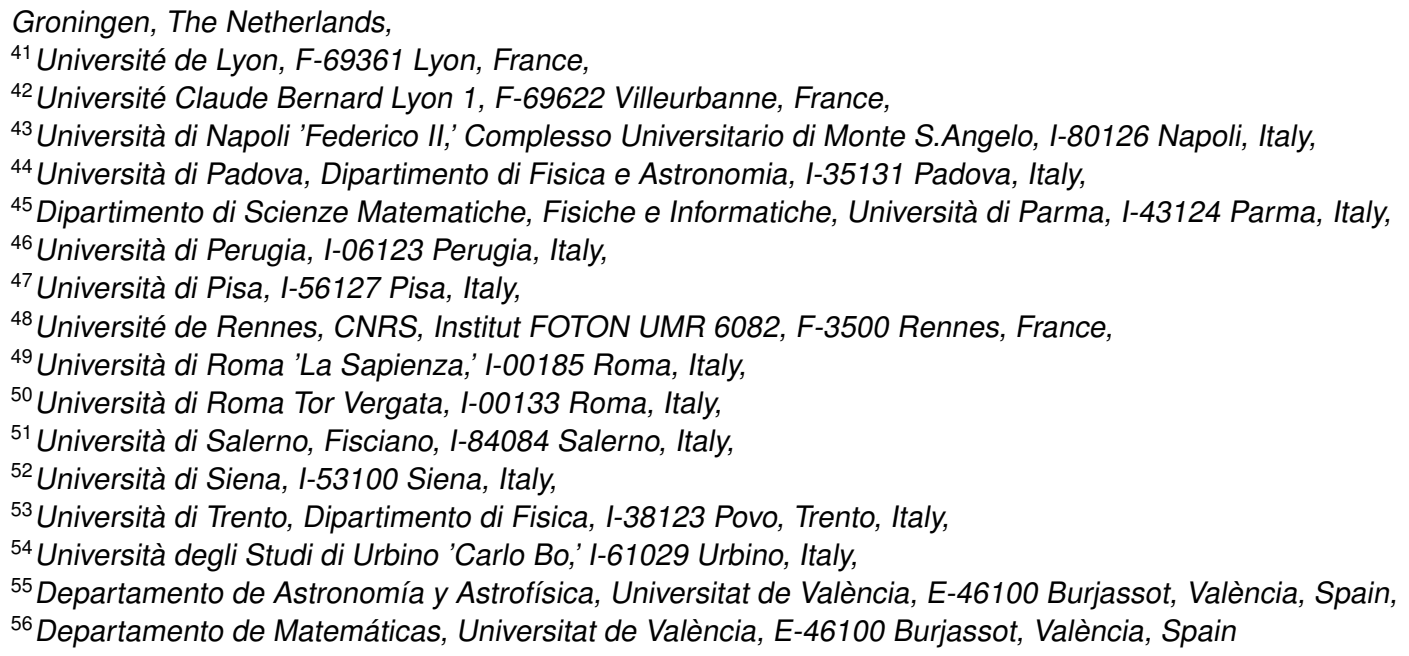

\begin{abstract}
The LIGO and the Virgo collaborations have recently announced the first detections of Gravitational Waves. Due to their weak amplitude, Gravitational Waves are expected to produce a very small effect on free-falling masses, which undergo a displacement of the order of $10^{-18} \mathrm{~m}$ for a $\mathrm{Km}$-scale mutual distance. This discovery showed that interferometric detectors are suitable to reveal such a feeble effect, and therefore represent a new tool for astronomy, astrophysics and cosmology in the understanding of the Universe.

To better reconstruct the position of the Gravitational Wave source and increase the signal-to-noise ratio of the events by means of multiple coincidence, a network of detectors is necessary. In the USA, the LIGO project has recently concluded its second Observation Run (O2) with a couple of twin 4 kilometer-long arms detectors which are placed in Washington State and Louisiana.

Advanced VIRGO (AdV) is a 3 kilometer-long arms second generation interferometer situated in Cascina, near Pisa in Italy. The installation of AdV has been completed in 2016, and the first commissioning phase allowed to get to the target early-stage sensitivity, which was sufficient to join LIGO in the $\mathrm{O} 2$ scientific run.

In this paper, the challenges of the commissioning of $\mathrm{AdV}$ will be presented, together with its current performances and future perspectives.

Finally, in the last paragraph the latest discoveries that occurred after the ICNFP 2017 conference will be also described.
\end{abstract}

\title{
1 Introduction
}

Gravitational Waves $(\mathrm{GW})$ are a perturbation of the flat space-time metrics due to the acceleration of masses. They are foreseen by Einstein's field equations of the General Relativity, which can be linearized in weak field approximation, giving rise to a wavy solution.

Given the quadrupolar moment tensor $Q_{\mu \nu}$ of the GW sources, the induced strain tensor $h_{\mu \nu}$ can be written as:

$$
h_{\mu v}=\frac{2 G}{r c^{4}}\left(\frac{d^{2} Q_{\mu v}}{d t^{2}}\right)
$$


where $r$ is the source distance, $G$ the Gravitational constant, and $c$ the speed of light. The GW amplitude is therefore proportional to the second time derivative of quadrupolar moment tensor through $G / c^{4} \approx 10^{-47} \mathrm{~N}^{-1}$. This number being very tiny, explains why only huge accelerated masses can give rise to detectable effects.

The typical strain amplitude for gravitational waves is expected to be of the order to $h \leq 10^{-21}$. Given two free-falling masses separated by a distance $L$, the effect induced by the GW can be estimated as:

$$
\delta L=\frac{h L}{2}
$$

which gives a $\delta L \approx 10^{-18} \mathrm{~m}$ for a kilometer-scale separation $L$ between masses.

The nature of GW polarization suggests that the induced variation is intrinsically differential. At the moment, the most sensitive instruments to measure such relative distance changes are Michelson interferometers.

In the interferometer, the masses are suspended to a chain of pendulums in order to reproduce the free falling condition for a frequency range higher than the pendulum resonance frequency. A gravitational wave will produce a differential elongation of the two arms, as shown in figure 1, which will be translated into a phase shift $\delta \phi$ at the output of the interferometer:

$$
\delta \phi=\frac{4 \pi}{\lambda} \delta L
$$

where $\lambda$ is the wavelength of the circulating light.

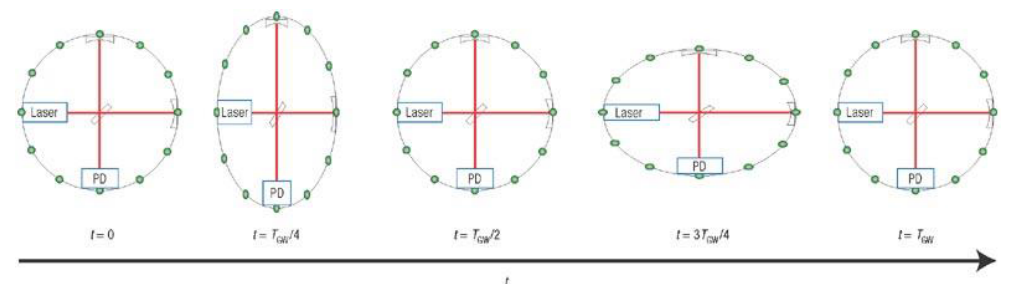

Figure 1. Effect on a Michelson interferometer of a gravitational wave propagating orthogonally to the plane of the page.

\section{From first to second generation detectors}

First generation of Gravitational Waves Interferometers such as Virgo [1], LIGO [2] and GEO600 [3] operated for about a decade from 2002 to 2011 , reaching a peak sensitivity down to $\tilde{h} \approx 10^{-22} / \sqrt{H z}$ and operating for long periods with a duty cycle of about $80 \%$.

No GW detection was performed with the first generation interferometers. However, besides demonstrating a reliable and promising technology, the Virgo and LIGO experiments provided an excellent knowledge of limiting noise sources.

The general approach adopted to improve the detectors sensitivity was to reduce the fundamental noise sources with a proper design, and to benefit of the acquired experience in operating the first generation instruments to control all technical noise sources.

The design sensitivity of the second generation detectors is one order of magnitude better than the previous generation, which corresponds to an increase of about a factor thousand in the observed volume. 

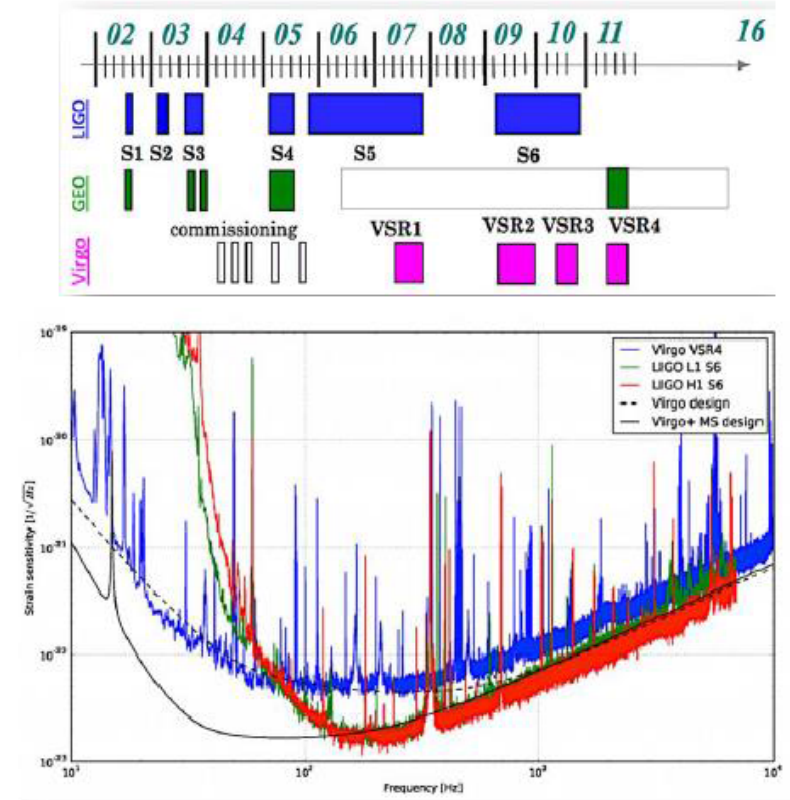

Figure 2. Top: Timeline of the Science Runs for the First generation of Gravitational Wave interferometric antennas. Bottom: First generation LIGO and Virgo strain sensitivities at their best, before the shutdown to allow the upgrade to second generation.

\section{The Advanced Virgo project}

The Advanced Virgo project takes advantage of the Virgo detector infrastructures, and is situated in Cascina, near Pisa (Italy). It is participated by six European countries, among which Italy and France (former founders of Virgo), The Netherlands, Hungary, Poland and Spain.

\subsection{Advanced Virgo configuration}

The optical scheme of Advanced Virgo is reported in figure 3. Four suspended mirrors, which form two Fabry-Pérot cavities along each 3-km arm of the Michelson interferometer, represent the test masses sensing the Gravitational Wave effect. The input laser beam, a CW Nd:YAG high power laser, is filtered by a Mode Cleaner cavity before being injected into the interferometer. A Power Recycling Mirror (PRM) is suspended along the laser path before the beam splitter mirror: it is used to recycle the power sent back towards the laser source, with the aim of further enhancing the circulating optical power.

The main difference between the optical schemes of first and second generation detectors is represented by the Signal Recycling Mirror (SRM), and therefore by the signal recycling cavity: depending on the cavity length and finesse tuning, it is possible to change the shape of the sensitivity curve and optimize it for different astrophysical sources. This kind of topology is called dual-recycled Fabry-Pérot Michelson interferometer [4].

Before reaching the output photodiode, the beam exiting the interferometer is further filtered by two 
mode cleaner cavities which cut out any residual spurious mode.

The Michelson interferometer is tuned in such a way that the two recombining beams interfere destructively. This working point is called dark fringe condition: for a good sensitivity, it is crucial not to introduce any asymmetry between the two arms which could spoil the interference condition.

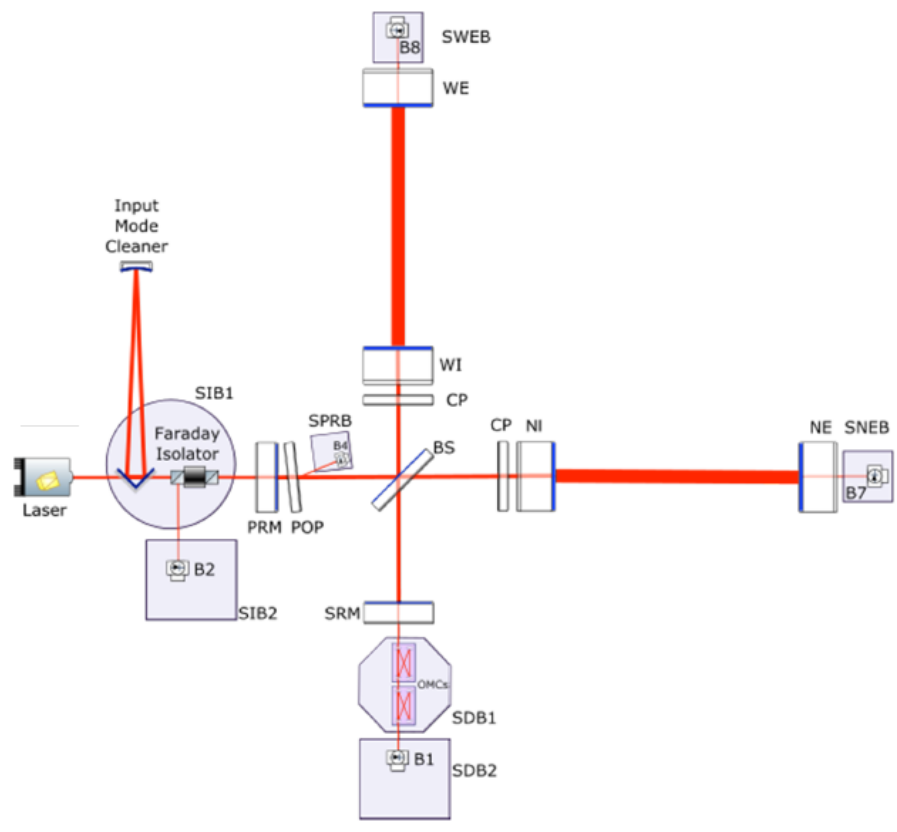

Figure 3. Advanced Virgo simplified optical scheme.

\subsection{Advanced Virgo sensitivity}

A typical figure of merit used to describe the sensitivity of a Gravitational Waves detector is given by the Binary-Neutron-Stars (BNS) Range, which is the volume-and-orientation-averaged distance at which a compact binary coalescence consisting of two 1.4 solar masses neutron stars gives a matched filter SNR of 8 in a single detector [6].

In figure 4 the spectral distribution of the main contributions to the $\mathrm{AdV}$ noise budget is reported. The sum of all these contribution corresponds to Advanced Virgo sensitivity, which extends from about 10 $\mathrm{Hz}$ to $10 \mathrm{kHz}$. The challenge of second generation detectors has consisted of evaluating and lowering as much as possible all these noise sources. This required large investments in infrastructures, a deep detector redesign, besides new technological developments.

\subsubsection{Low frequency region $(10 \mathrm{~Hz}-40 \mathrm{~Hz})$}

Among fundamental noises, in the low frequency range $(10 \mathrm{~Hz}-40 \mathrm{~Hz})$ the main offenders are represented by Newtonian noise, seismic noise and suspensions thermal noise. The Newtonian noise (or 


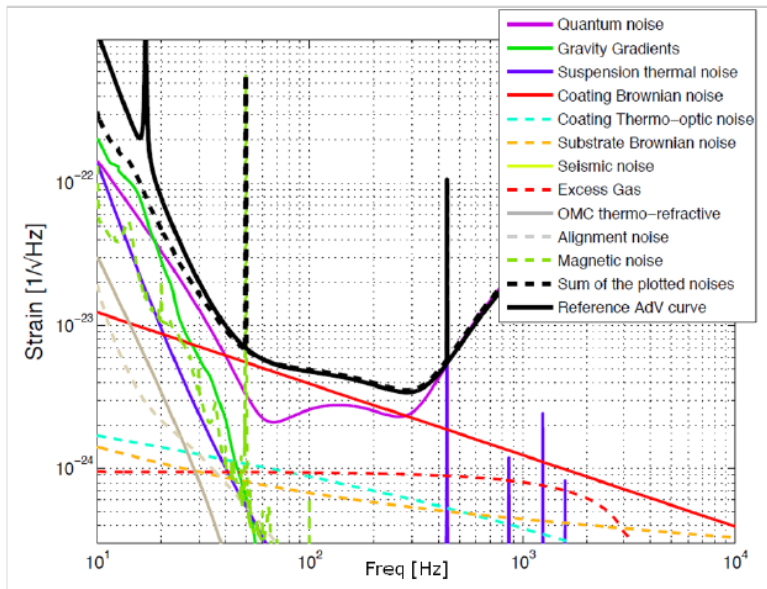

Figure 4. Advanced Virgo strain sensitivity for a detuned dual recycled configuration with $125 \mathrm{~W}$ of input power (solid black line). The main noise sources limiting at different frequency ranges are also reported. Plot from Advanced Virgo Technical Design Report [5].

gravity gradient noise) is caused by the fluctuation of the local gravitational field due to seismic waves in the ground that change the mass density distribution. However, this is dominant in the frequency band below $5 \mathrm{~Hz}$, and is overcome by seismic noise in the frequency band above $10 \mathrm{~Hz}$. Seismic noise is due to ground vibrations coupling to the interferometer mirrors through their suspension system, and represent the most relevant sensitivity limit at low frequency. Seismic noise is usually modeled by assuming a white acceleration noise above $10 \mathrm{mHz}$, corresponding to a $1 / f^{2}$ strain equivalent noise spectrum. This noise source is mitigated by suspending the mirrors with a complex isolation system called Superattenuator (figure 5), which is made of a chain of $7.5 \mathrm{~m}$ long hybrid (passive-active) mechanical filters, able to low-pass vibrations in 6 degrees of freedom above their resonance frequency, achieving a reduction of the seismic noise by about 12 orders of magnitude. The isolation performance of the Superattenuators implemented during Virgo have been demonstrated to be compliant with the requirements of Advanced Virgo, and only minor changes were brought to the suspension design.

The only noise source which is not mitigated in this frequency region and which therefore represents a fundamental limit to the sensitivity is the suspension thermal noise. According to fluctuationdissipation theorem, any mechanical oscillator is affected by a motion of thermal origin directly related to its thermodynamic temperature. The mirrors and their suspensions are examples of these mechanical oscillators. As a consequence, their position is affected by this thermal vibration, which can be dumped, for instance, by decreasing its temperature.

\subsubsection{Mid-frequency region $(40 \mathrm{~Hz}-300 \mathrm{~Hz})$}

The mid-frequency range $(40 \mathrm{~Hz}-300 \mathrm{~Hz})$ is mainly dominated by the thermal noise. It has two main contributions: first, the violin modes of the suspension wires and, second, the mirror coating excited by the thermal environment. In particular, this latter is due to several effects, such as Brownian motion, thermo-elastic and thermo-refractive fluctuations in the mirror bulk.

The mitigation of the first contribution is foreseen to be achieved by installing monolithic suspensions, 


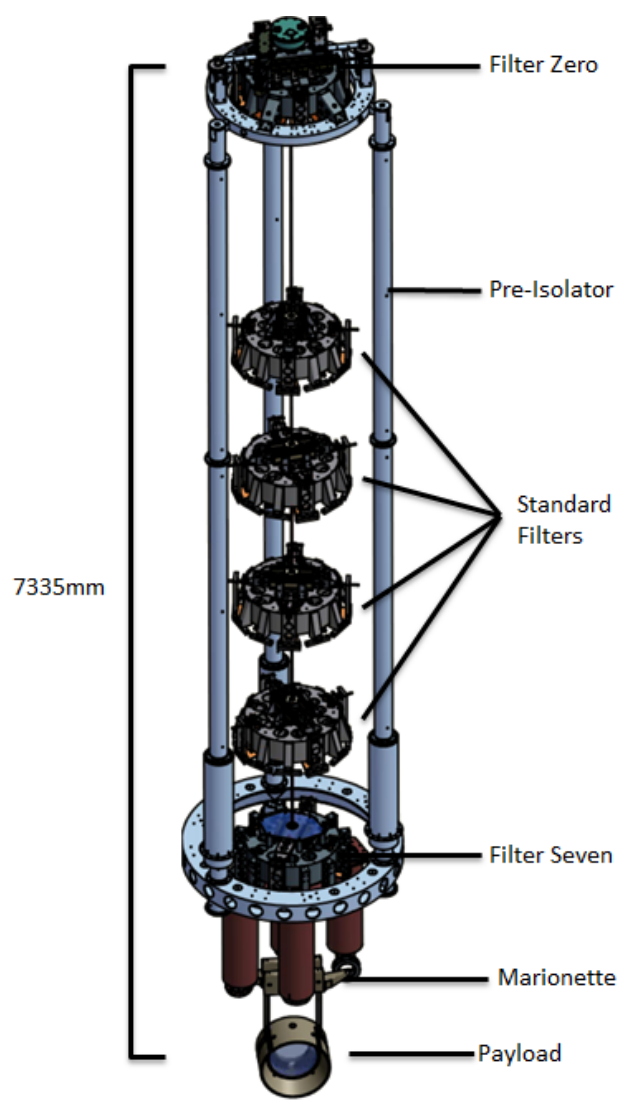

Figure 5. The Virgo Superattenuator. The system is formed by a chain of passive mechanical filters with resonance frequency $f_{0} \approx 0.6 \mathrm{~Hz}$, each providing a seismic noise attenuation by a factor $\left(f / f_{0}\right)^{2}$, a three legs structure based on the working principle of an Inverted Pendulum and a Payload as last stage.

that is to hang up the test masses by means of fused silica fibers, whose intrinsic dissipation is about three orders of magnitude lower than steel. On the other hand, the mitigation of coating vibrations mainly relies on enlarging the beam size on the Test Masses, in such a way to reduce the beam intensity impinging on the mirror surface.

Besides them, one of the worse offending noise at low-mid frequency range is due to stray light, such as ghost beams and scattered light, probing vibrations of mechanical structures and then recombining to the interferometer main beam. The mitigation of this noise source passes through two steps: on the one hand, the installation of new baffles of different shapes and materials, able to intercept and dump most of the predicted stray light, and secondly an improved mirror planarity, with an average RMS lower than $0.5 \mathrm{~nm}$ for the test masses. In addition, a new and more powerful system of thermal compensation (TCS) has been designed and installed, to actively correct for thermally induced deformations and cold aberrations which could highlight and put into resonance unwanted modes, therefore preventing the perfect recombination of the beams at the interferometer output. Test masses are all equipped with ring heaters to correct for radius of curvature discrepancies, and $\mathrm{CO}_{2}$ lasers can project different patterns onto the dedicated optics (compensation plates) to provide compensations 
of mirror defects. Appropriate sensors such as Hartmann wavefront sensors and phase cameras have been installed to provide monitor signals for these aberrations.

\subsection{High-frequency region $(300 \mathrm{~Hz}-10 \mathrm{kHz})$}

In the high frequency range $(300 \mathrm{~Hz}-10 \mathrm{kHz})$ the sensitivity is dominated by the quantum noise. This fundamental noise source has two main contributions: on the one hand, the statistical fluctuation of the number of detected photons (shot noise), which introduces a strain equivalent white noise spectrum:

$$
\tilde{h}_{S N}=\frac{1}{L} \sqrt{\frac{\hbar c \lambda}{2 \pi P}}
$$

where $\mathrm{L}$ is the interferometer arms length and $\mathrm{P}$ is the input laser power. This white noise is actually multiplied by the optical response of the interferometer, due to the lowpass filtering action of the Fabry-Pérot cavities in the arms, with a cutoff frequency $f_{c} \approx 55 \mathrm{~Hz}$. On the other hand, the quantum noise manifests itself as radiation pressure fluctuations on the test masses which, in turn, produce a $1 / f^{2}$ strain equivalent noise spectrum:

$$
\tilde{h}_{R P}=\frac{1}{2 \pi f^{2} M L} \sqrt{\frac{8 \pi \hbar P}{\lambda c}}
$$

Here $\mathrm{M}$ is the mass of each mirror. The two terms scale in opposite way with input power.

In order to mitigate this noise, Advanced Virgo arm Fabry-Perot cavities have an increased Finesse and the injected laser power is designed to be about 16 times larger than in Virgo. Radiation pressure noise has been mitigated by increasing the weight of the test masses, bringing it from $21 \mathrm{~kg}$ to $42 \mathrm{~kg}$ (figure 6).

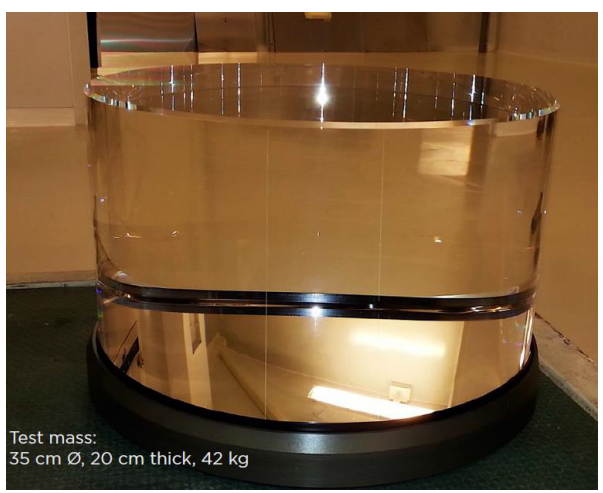

Figure 6. Advanced Virgo test mass. The Fused Silica substrate $0.35 \mathrm{~m}$ diameter is $0.2 \mathrm{~m}$ thick, for a total weight of $42 \mathrm{~kg}$.

\section{From installation to scientific run}

The Virgo detector was de-commissioned starting from the end of 2011, the integration of Adv-Virgo detector started in 2012 and was completed during 2016. This phase was followed by a very intensive 
Commissioning activity, which brought the second generation interferometer to a good operating point, compliant with an early stage target sensitivity.

\subsection{Integration issues}

The integration process way slowed down by many issues, mainly concerning the Superattenuator blades, the core optics quality and the monolithic suspensions integration.

Each mechanical filter of the super-attenuator is provided with a set of triangular cantilever spring blades which provide a vertical attenuation. This hardware worked for more than 10 years without relevant issues. However, during the integration of the new payloads, some of the maraging steel blades were found faulty - subsequent investigations showed because of embrittlement due to Hydrogen contamination during the machining process. Because of this failure, an inspection of the status of all the blades was performed, and $40 \%$ of all of them (as a precaution) was replaced.

After their installation, one of the core optics surface was found to be affected by a round shaped scratch in the middle. The investigation on the damage suggested a re-opening of the vacuum chamber and a replacement of the optic with a spare one.

Finally, one of the most worrisome issues was the monolithic suspension failure. Monolithic suspensions were already successfully demonstrated during the Virgo+ phase, so no issues were expected from this side but, quite surprisingly, repeated breaking of monolithic suspensions under vacuum occurred during installations on 2015-2016. After wide investigations performed to find the causes of failure, the basic mechanism of fiber breaking under vacuum was eventually identified: fast dust particles hitting the fiber and producing fractures, which evolved with different rate until a full break. Temporary solution, driven mainly by schedule considerations, was to replace them back to steel wires for payloads. Sensitivity with steel wires has been calculated to be still compatible with the goal for the early stage of Advanced Virgo. The origin of the dust particles was found to be a scroll pump, whose replacement is mandatory in view of the next upgrade phase prior to the third joint observation run, which foresees the installation the monolithic suspension to mitigate thermal noise. In the same line, the payloads will be equipped with a special shield protecting the glass fibers from possible residual particles.

\subsection{Commissioning}

The commissioning of the whole interferometer started at the end of October 2016. In order to join the second part of $\mathrm{O} 2$ scheduled for August 2017, the commissioning activity had to exploit this left only ten months to go from scratch to a decent sensitivity for Advanced Virgo.

The first achieved milestone was the one hour long lock of the interferometer at what is called dark fringe working point: this happened after six months from the beginning of the commissioning, on March 2017. Only two months after the commissioning brought the interferometer up to the point of being operated at its working point for few days. Less than a month later, an engineering run (ER11) was held in coincidence with aLIGO: first part from 16th to 19th with a BNS range varying from 5 to $9 \mathrm{Mpc}$, and a duty cycle of $70 \%$, second part from 23 rd to 26 th with a BNS range of $8-9 \mathrm{Mpc}$, duty cycle of about $80 \%$.

Despite of the satisfactory durty cycle, the interferometer sensitivity was still too low to really hope to join $\mathrm{O} 2$ on time. So, after ER11 an even more intense campaign of noise investigation was launched to achieve a sensitivity allowing to contribute to the science of aLIGO observing run. This phase was characterized by investigation on stray light and subsequent mitigation of scattering sources (where 


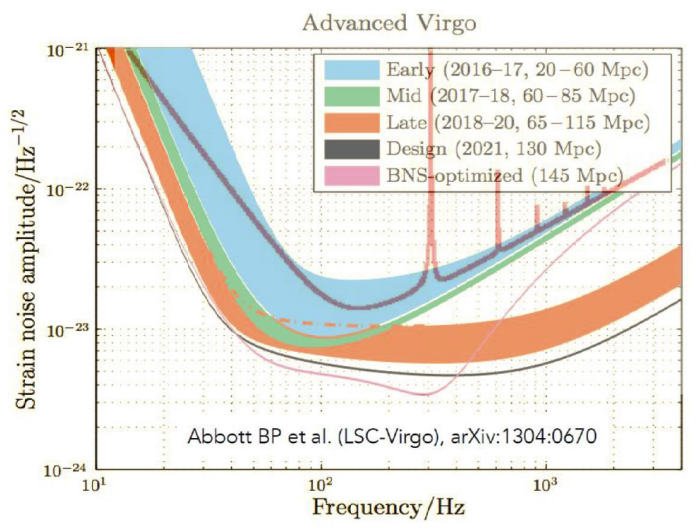

Figure 7. Advanced Virgo target strain sensitivity as a function of frequency. The binary neutron-star (BNS) range, the average distance to which these signals could be detected, is given in megaparsec. Current notions of the progression of sensitivity are given for early, mid and late commissioning phases, as well as the final design sensitivity target and the BNS-optimized sensitivity. Plot from [7]. The brown curve represents the sensitivity reached with the steel wires, which is still compatible with the goal for the early phase.

possible), noise injections (magnetic, acoustic, seismic, etc.) and switch-off tests to understand coupling from environment and devices to the interferometer. Furthermore, some room for optimization of Data Acquisition pipeline and read-out was still left, and for lock robustness improvement (alignment, loop accuracy, etc.) as well.

After this uninterrupted work, the BNS range reached 20Mpc just a couple of weeks before joining $\mathrm{O} 2$, to reach peaks of $30 \mathrm{Mpc}$ during the run itself, which was enough to contribute to science data as part of the network of three interferometers (figure 8).

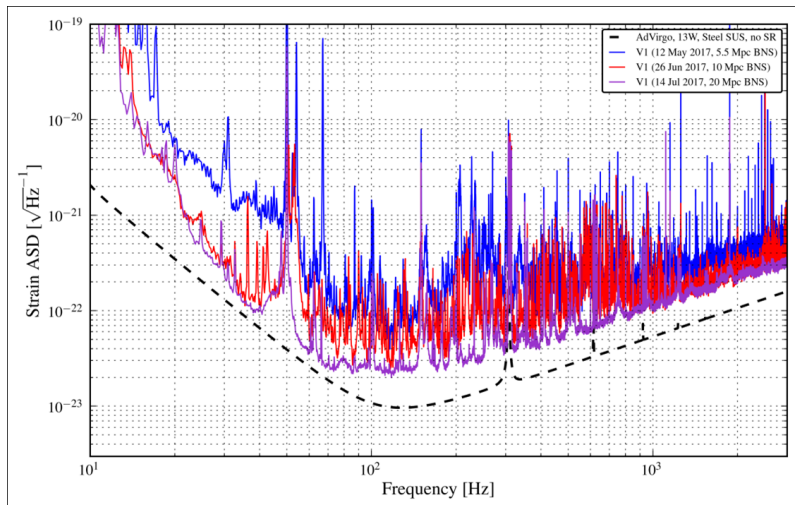

Figure 8. Sensitivity evolution from mid-May to mid-July. Thanks to the noise hunting activity, to the improvement of loop robustness, and to a further optimization of Data Acquisition process, the BNS range has increased from 5 to $20 \mathrm{Mpc}$. 


\section{Second generation detectors joint observation run}

\subsection{Detector performance}

August $1^{\text {st }}$ marks the beginning of the first joint Second Generation Detectors run, which sees the two LIGO detectors and the Virgo detector taking data together.

Figure 9 shows the sensitivity of the two aLIGO detectors (in light blue and in red), of the Advanced Virgo detector (purple), and of the GEO600 detector, an interferometer situated in Hannover (Germany), whose sensitivity becomes comparable to the detector network in the frequency region above few kHz.

Advanced Virgo has shown a great stability during O2, reaching a very high duty cycle (figure 10) even higher than the two aLIGO detectors, with locking segments lasting up until $\tilde{70}$ hours.

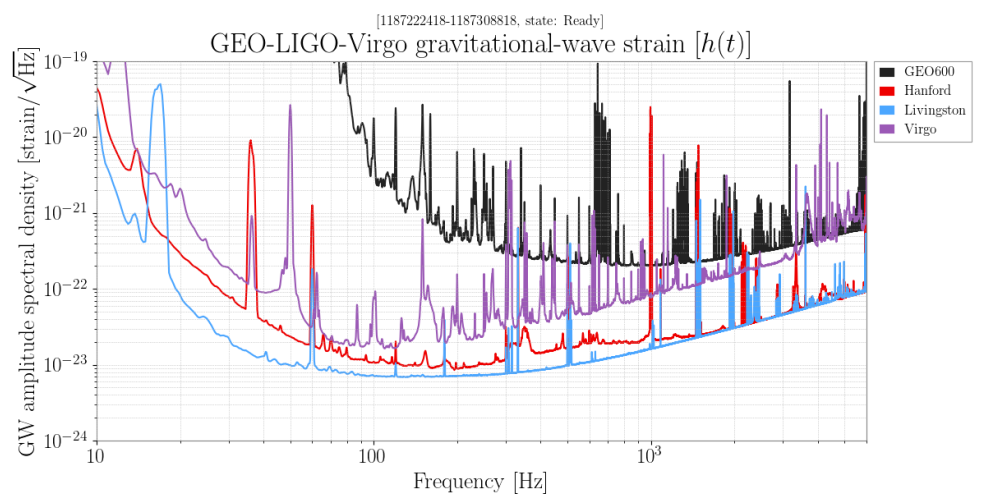

Figure 9. Sensitivity of the Second Generation Detectors during the second Scientific Run O2. The sensitivity of aLIGO Livingston and aLIGO Hanford are shown in light blue and red, respectively. In purple, Advanced Virgo sensitivity. In black, finally, the sensitivity of the German interferometer GEO600, which is comparable to the Advanced Virgo sensitivity in the frequency range above few $\mathrm{kHz}$.

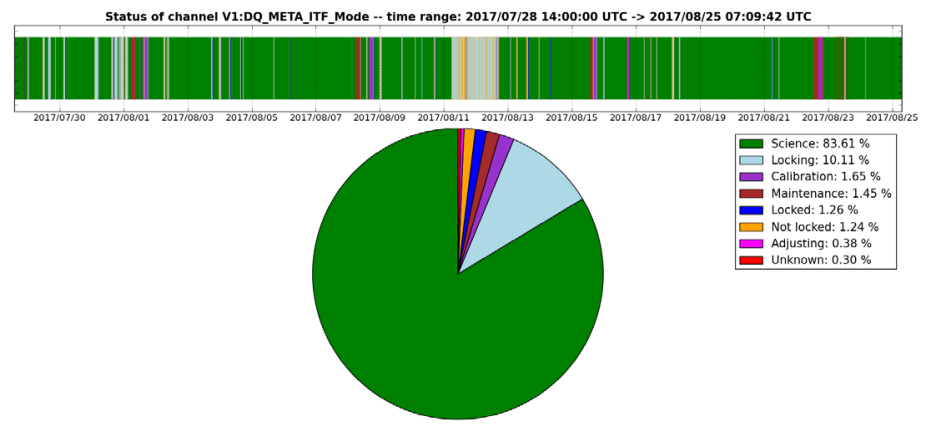

Figure 10. Advanced Virgo duty cycle during O2. The longest lock segment lasted about 69 hours. The detector was operated in Science Mode for about $83 \%$ of the total time. 


\subsection{First detection from LIGO-Virgo detectors}

The joint observation run with aLIGO and Advanced Virgo has been concluded few days after the ICNFP conference 2017, so what was reported in the talk didn't account for the analysis of the collected data. However, it is worth mentioning the results of the analysis which became public after the end of the conference.

Advanced Virgo, as part of the network, detected at least two GW signals. Thanks to the presence of the third detector, it was possible to significantly restrict the uncertainty area on the celestial sphere associated with gravitational waves signals. The first detected event was produced by a Binary Black Holes coalescing system, as for the previous events detected by the aLIGO detectors. This event is referred to as GW170814 and is the first event ever detected by the Virgo interferometer [8], in triple coincidence with the twin LIGO detectors. A plot of the detected signal as seen in the interferometer time domain data is shown in figure 11. GW170814 demonstrates the potential of a 3-detector network, both in terms of localization of a source in the sky and in terms of the testing of Einstein's theory of General Relativity.

During the same science run another event was also detected in triple coincidence. The signal called GW170817 was instead produced by a couple of coalescing Neutron Stars, the first event ever observed from this kind of source. Thanks to the better localization (see figure 12), the observation of an electromagnetic counterpart of the GW source was possible. This stated the beginning of the Multi-messenger Astronomy [9], [10].
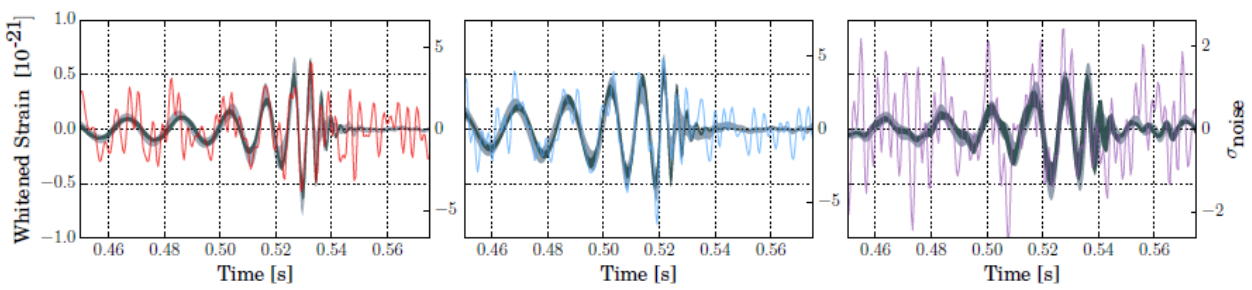

Figure 11. Triple coincidence of the Binary Black Hole signal detected on August $14^{\text {th }} 2017$. From left to right, time series of the LIGO Hanford, LIGO Livingston and Virgo observatory superimposed to the reconstructed signal.

\section{Conclusions}

After more than 50 years of experimental research the first detection of gravitational waves has opened a new era in the astronomy and astrophysics. Thanks to an improved design, the Second Generation Detectors sensitivity is foreseen to be enhanced of about a factor 10 on the whole measurement band with respect to the previous sensitivity. After a 4 years long installation phase, followed by an intense commissioning activity, on August $1^{\text {st }}$ the Adv-Virgo detector has joined the twins aLIGO detectors in the second Observation Run $(\mathrm{O} 2)$, and the three interferometer collected data together for about one month. A deep analysis of the data has shown the presence of at least two signals detected in triple coincidence, one of them being produced by a Binary Neutron Stars System. Thanks to the improved accuracy in the source localization due to the presence of the third detector, it was possible to observe also the electromagnetic counterpart associated to the Gravitational Wave event. This has marked the beginning of the Multi-messenger Astronomy. 


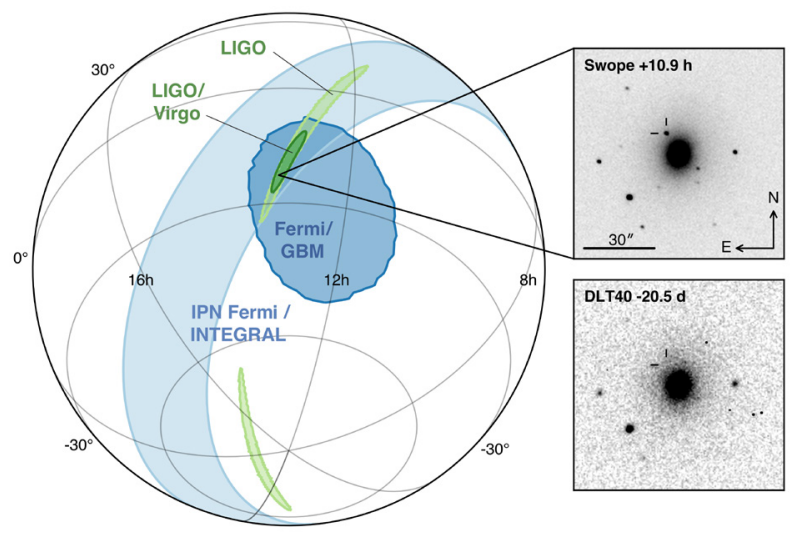

Figure 12. Sky localization of the GW170817 Gravitational Wave event. Thanks to the Advanced Virgo contribution, the interesting area could be reduced to a sky region of $28 \mathrm{deg}^{2}$. This event served as a trigger for electromagnetic telescopes, which were able to detect the optical counterpart of the GW source in the Galaxy NGC4993. In the pictures on the right, NGC4993 before (bottom) and after (top) the detection of GW170817 by aLIGO and Advanced Virgo.

\section{References}

[1] Accadia T et al., JINST 7, P03012 (2012).

[2] Abbott B P et al., Observatory Rep. Prog. Phys. 72, 076901 (2009).

[3] Wilke B et al., Class. Quantum Grav. 19, 1377 (2002).

[4] Acernese F et al., Class. Quantum Grav. 32, 024001 (2014).

[5] The Virgo collaboration, Virgo Technical documentation system, VIR-0128A-12 https://tds.virgo-gw.eu/ql/?c=8940

[6] L. S. Finn and D. F. Cherno, Phys. Rev. D 47, 2198-2219 (1993).

[7] Abbott B P et al., Living Rev. Relativity, 19, (2016), 1.

[8] Abbott B P et al., Phys. Rev. Lett. 119, 141101 (2017).

[9] Abbot B P et al., Phys. Rev: Lett, 119, 161101 (2017).

[10] Abbott B P et al., ApJL 848, L12 (2017). 